\title{
Existence for Elliptic Equation Involving Decaying Cylindrical Potentials with Subcritical and Critical Exponent
}

\author{
Mohammed El Mokhtar Ould El Mokhtar \\ Department of Mathematics, College of Science, Qassim University, P.O. Box 6644, Buraidah 51452, Saudi Arabia \\ Correspondence should be addressed to Mohammed El Mokhtar Ould El Mokhtar; med.mokhtar66@yahoo.fr
}

Received 4 July 2015; Accepted 13 October 2015

Academic Editor: Gershon Wolansky

Copyright ( 2015 Mohammed El Mokhtar Ould El Mokhtar. This is an open access article distributed under the Creative Commons Attribution License, which permits unrestricted use, distribution, and reproduction in any medium, provided the original work is properly cited.

We consider the existence of nontrivial solutions to elliptic equations with decaying cylindrical potentials and subcritical exponent. We will obtain a local minimizer by using Ekeland's variational principle.

\section{Introduction}

In this paper, we study the existence of nontrivial solutions of the following problem:

$$
\begin{array}{r}
-\Delta u-\mu|y|^{-2} u=|y|^{-a \gamma}|u|^{\gamma-2} u(1+\lambda g(x)) \\
\quad \text { in } \mathbb{R}^{N}, y \neq 0, u>0,
\end{array}
$$

where $y \in \mathbb{R}^{k}$, and let $k$ and $N$ be integers such that $N \geq 3$ and $k$ belongs to $\{1, \ldots, N\} .2^{*}=2 N /(N-2)$ is the critical Sobolev exponent, $\gamma \leq 2^{*}, 0 \leq a<1, g$ is a continuous function on $\mathbb{R}^{N}$, and $\lambda$ and $\mu$ are parameters which we will specify later.

We denote point $x$ in $\mathbb{R}^{N}$ by the pair $(y, z) \in \mathbb{R}^{k} \times \mathbb{R}^{N-k}$, $\mathscr{D}_{0}^{1,2}=\mathscr{D}_{0}^{1,2}\left(\left(\mathbb{R}^{k} \backslash\{0\}\right) \times \mathbb{R}^{N-k}\right)$, and $\mathscr{H}_{\mu}=\mathscr{H}_{\mu}\left(\left(\mathbb{R}^{k} \backslash\{0\}\right) \times\right.$ $\left.\mathbb{R}^{N-k}\right)$, the closure of $C_{0}^{\infty}\left(\left(\mathbb{R}^{k} \backslash\{0\}\right) \times \mathbb{R}^{N-k}\right)$ with respect to the norms

$$
\begin{aligned}
\|u\| & =\left(\int_{\mathbb{R}^{N}}|\nabla u|^{2}\right)^{1 / 2} \\
\|u\|_{\mu} & =\left(\int_{\mathbb{R}^{N}}\left(|\nabla u|^{2}-\mu|y|^{-2}|u|^{2}\right) d x\right)^{1 / 2}
\end{aligned}
$$

with $\mu<\bar{\mu}_{k}=((k-2) / 2)^{2}$ for $k \neq 2$.

From the Hardy inequality, it is easy to see that the norm $\|u\|_{\mu}$ is equivalent to $\|u\|$.
We define the weighted Sobolev space $\mathscr{D}:=\mathscr{H}_{\mu} \cap$ $L^{\gamma}\left(\mathbb{R}^{N},|y|^{-b} d x\right) \cap L^{2}\left(\mathbb{R}^{N},|y|^{-2} d x\right)$ with $b=a \gamma$, which is a Banach space with respect to the norm defined by $\mathcal{N}(u):=$ $\|u\|_{\mu}+\left(\int_{\mathbb{R}^{N}}|y|^{-b}|u|^{\gamma} d x\right)^{1 / \gamma}$.

My motivation of this study is the fact that such equations arise in the search for solitary waves of nonlinear evolution equations of the Schrödinger or Klein-Gordon type (cf. [1-3]). Roughly speaking, a solitary wave is a nonsingular solution which travels as a localized packet in such a way that the physical quantities corresponding to the invariances of the equation are finite and conserved in time. Accordingly, a solitary wave preserves intrinsic properties of particles such as the energy, the angular momentum, and the charge, whose finiteness is strictly related to the finiteness of the $L^{2}$-norm. Owing to their particle-like behavior, solitary waves can be regarded as a model for extended particles and they arise in many problems of mathematical physics, such as classical and quantum field theory, nonlinear optics, fluid mechanics, and plasma physics (see, e.g., [4]).

Several existence and nonexistence results are available in the case $k=N$, and we quote, for example, [5-7] and the references therein. When $\mu=0, g(x) \equiv 1$; problem $\left(\mathscr{P}_{\lambda, \mu}\right)$ has been studied in the famous papers by Brézis and Nirenberg [8] and Xuan [9] which consider the existence and nonexistence of nontrivial solutions to quasilinear BrézisNirenberg-type problems with singular weights.

Concerning the existence result in the case $k<N$, we cite $[10,11]$ and the references therein. As noticed in [10], for 
$\mu<0$ and $a=0$, Badiale and Rolando have considered the problem $\left(\mathscr{P}_{0, \mu}\right)$. They established the existence of nontrivial nonnegative radial solution when $\beta \in(0,2)$ and $\gamma \in\left(2_{\beta}, 2^{*}\right)$ or $\beta \in(2,+\infty)$ and $\gamma \in\left(2^{*}, 2_{\beta}\right)$; in addition, if the function $f(u)=|u|^{\gamma-1} u$ is odd, then $\left(\mathscr{P}_{0, \mu}\right)$ has infinitely many radial solutions. In [5], Badiale et al. proved the nonexistence of nonzero classical solutions when $k \leq N$ and the pair $(\beta, \gamma)$ belongs to the light gray region. That is, $(\beta, \gamma) \in \mathscr{A}=\mathscr{A}_{1} \cup$ $\mathscr{A}_{2} \cup \mathscr{A}_{3}$, where

$$
\begin{aligned}
& \mathscr{A}_{1}:=\left\{(\beta, \gamma) \in \mathbb{R}^{2}: \beta \in(0,2), \gamma \notin\left(2,2^{*}\right), \gamma \geq 2\right\} \backslash\left\{\left(2,2^{*}\right)\right\}, \\
& \mathscr{A}_{2}:=\left\{(\beta, \gamma) \in \mathbb{R}^{2}: \beta \in(2, N), \gamma \notin\left(2^{*}, 2_{\beta}\right), \gamma \geq 2\right\}, \\
& \mathscr{A}_{3}:=\left\{(\beta, \gamma) \in \mathbb{R}^{2}: \beta \in[N,+\infty), \gamma \in\left[2,2^{*}\right]\right\} .
\end{aligned}
$$

Since our approach is variational, we define the functional $I_{\lambda, \mu}$ on $\mathscr{D}$ by

$$
\begin{aligned}
I_{\lambda, \mu}(u):= & \left(\frac{1}{2}\right)\|u\|_{\mu}^{2} \\
& -\left(\frac{1}{\gamma}\right) \int_{\mathbb{R}^{N}}|y|^{-b}|u|^{\gamma}(1+\lambda g(x)) d x .
\end{aligned}
$$

We say that $u \in \mathscr{D}$ is a weak solution of the problem $\left(\mathscr{P}_{\lambda, \mu}\right)$ if it is a nontrivial nonnegative function and satisfies

$$
\begin{aligned}
& \left\langle I_{\lambda, \mu}^{\prime}(u), v\right\rangle:=\int_{\mathbb{R}^{N}}\left(\nabla u \nabla v-\mu|y|^{-2} u v\right. \\
& \left.\quad-|y|^{-b}|u|^{\gamma-2} u v(1+\lambda g(x))\right)=0, \quad \text { for } v \in \mathscr{D} .
\end{aligned}
$$

Throughout this work, we consider the following regions $\mathscr{R}_{1}$, $\mathscr{R}_{2}$, such that

$$
\begin{aligned}
& \mathscr{R}_{1}:=\left\{(2, \gamma) \in \mathbb{R}^{2}: \gamma \in\left(2_{2-2 a}, 2^{*}\right)\right\}, \\
& \mathscr{R}_{2}:=\left\{(2, \gamma) \in \mathbb{R}^{2}: \gamma \in\left(2,2_{2-2 a}\right)\right\}
\end{aligned}
$$

with $2_{2-2 a}=2 N /(N-(2-2 a))$.

Concerning the perturbation $g$, we assume

$$
\begin{gathered}
g \in L^{\infty}\left(\mathbb{R}^{N}\right), \\
g(x)>0 \quad \forall x \in \mathbb{R}^{N} .
\end{gathered}
$$

In our work, we prove the existence of at least one critical point of $I_{\lambda, \mu}$ by Ekeland's variational principle in [12].

We will state our main result.

Theorem 1. Assume that $2<k \leq N, \mu<\bar{\mu}_{k}, 0<a<1$, and (G) hold.

If $(2, \gamma) \in \mathscr{R}_{1} \cup \mathscr{R}_{2}$, then there exists $\Lambda^{*}>0$ such that the problem $\left(\mathscr{P}_{\lambda, \mu}\right)$ has at least one nontrivial solution for any $\lambda>\Lambda^{*}$.

This paper is organized as follows. In Section 2, we give some preliminaries. Section 3 is devoted to the proof of Theorem 1.

\section{Preliminaries}

We list here a few integrals inequalities. The first inequality that we need is the weighted Hardy inequality [13]

$$
\bar{\mu}_{k} \int_{\mathbb{R}^{N}}|y|^{-2} v^{2} d x \leq \int_{\mathbb{R}^{N}}|\nabla v|^{2} d x, \quad \forall v \in \mathscr{H}_{\mu} .
$$

The starting point for studying $\left(\mathscr{P}_{\lambda, \mu}\right)$ is the Hardy-SobolevMaz'ya inequality that is peculiar to the cylindrical case $k<N$ and that was proved by Gazzini and Musina in [14]. It states that there exists positive constant $C_{\gamma}$ such that

$$
C_{\gamma}\left(\int_{\mathbb{R}^{N}}|v|^{\gamma} d x\right)^{2 / \gamma} \leq \int_{\mathbb{R}^{N}}\left(|\nabla v|^{2}-\mu|y|^{-2} v^{2}\right) d x,
$$

for $\mu=0$; equation of $\left(\mathscr{P}_{\lambda, \mu}\right)$ is related to a family of inequalities given by Caffarelli et al. [15], for any $v \in C_{c}^{\infty}\left(\left(\mathbb{R}^{k} \backslash\right.\right.$ $\left.\{0\}) \times \mathbb{R}^{N-k}\right)$. The embedding $\mathscr{H}_{\mu} \hookrightarrow L^{\gamma}\left(\mathbb{R}^{N},|y|^{-b} d x\right)$ is compact, where $b=a \gamma$ and $L^{\gamma}\left(\mathbb{R}^{N},|y|^{-b} d x\right)$ is the weighted $L^{\gamma}$ space with respect to the norm

$$
|u|_{\gamma, b}^{2}=\left(\int_{\mathbb{R}^{N}}|y|^{-b}|v|^{\gamma} d x\right)^{2 / \gamma}
$$

Definition 2. Assume $2 \leq k<N, 0<\mu \leq \bar{\mu}_{k}$, and $2<\gamma<2^{*}$. Then, the infimum $S_{\mu, \gamma}$ defined by

$$
S_{\mu, \gamma}=S_{\mu, \gamma}(k, \gamma):=\inf _{v \in \mathscr{D} \backslash\{0\}} \frac{\int_{\mathbb{R}^{N}}\left(|\nabla v|^{2}-\mu|y|^{-2} v^{2}\right) d x}{\left(\int_{\mathbb{R}^{N}}|y|^{-b}|v|^{\gamma} d x\right)^{2 / \gamma}}
$$

is achieved on $\mathscr{H}_{\mu}$.

Lemma 3. Let $\left(u_{n}\right) \subset \mathscr{D}$ be a Palais-Smale sequence $\left((P S)_{\delta}\right.$ for short) of $I_{\lambda, \mu}$ such that

$$
\begin{array}{r}
I_{\lambda, \mu}\left(u_{n}\right) \longrightarrow \delta, \\
I_{\beta, \lambda, \mu}^{\prime}\left(u_{n}\right) \longrightarrow 0
\end{array}
$$

$$
\text { in } \mathscr{D}^{\prime}(\text { dual of } \mathscr{D}) \text { as } n \longrightarrow \infty \text {, }
$$

for some $\delta \in \mathbb{R}$. Then, $u_{n} \rightarrow u$ in $\mathscr{D}$ and $I_{\beta, \lambda, \mu}^{\prime}(u)=0$.

Proof. From (10), we have

$$
\begin{aligned}
& \left(\frac{1}{2}\right)\left\|u_{n}\right\|_{\mu}^{2}-\left(\frac{1}{\gamma}\right) \int_{\mathbb{R}^{N}}|y|^{-b}\left|u_{n}\right|^{\gamma}(1+\lambda g(x)) d x \\
& =\delta+o_{n}(1) \\
& \left\|u_{n}\right\|_{\mu}^{2}-\int_{\mathbb{R}^{N}}|y|^{-b}\left|u_{n}\right|^{\gamma}(1+\lambda g(x)) d x=o_{n}(1),
\end{aligned}
$$

for $n$ large,

where $o_{n}(1)$ denotes $o_{n}(1) \rightarrow 0$ as $n \rightarrow \infty$. Then,

$$
\begin{aligned}
\delta+o_{n}(1) & =I_{\lambda, \mu}\left(u_{n}\right)-\left(\frac{1}{\gamma}\right)\left\langle I_{\beta, \lambda, \mu}^{\prime}\left(u_{n}\right), u_{n}\right\rangle \\
& =\left(\frac{(\gamma-2)}{2 \gamma}\right)\left\|u_{n}\right\|_{\mu}^{2},
\end{aligned}
$$


and $\left(u_{n}\right)$ is bounded in $\mathscr{D}$. Going if necessary to a subsequence, we can assume that there exists $u \in \mathscr{D}$ such that

$$
\begin{aligned}
& u_{n} \rightarrow u \quad \text { in } \mathscr{D}, \\
& u_{n} \rightarrow u \quad \text { in } L^{\gamma}\left(\mathbb{R}^{N},|y|^{-b} d x\right), \\
& u_{n} \rightarrow u \quad \text { a.e. in } \mathbb{R}^{N} .
\end{aligned}
$$

Consequently, we get, for all $v \in C_{0}^{\infty}\left(\left(\mathbb{R}^{k} \backslash\{0\}\right) \times \mathbb{R}^{N-k}\right)$,

$$
\begin{aligned}
& \int_{\mathbb{R}^{N}}\left(\nabla u \nabla v-\mu|y|^{-2} u v\right. \\
& \left.-|y|^{-b}|u|^{\gamma-2} u v(1+\lambda g(x))\right)=0,
\end{aligned}
$$

which means that

$$
I_{\beta, \lambda, \mu}^{\prime}(u)=0
$$

\section{Existence Result}

Firstly, we require the following lemmas.

Lemma 4. Let $\left(u_{n}\right) \subset \mathscr{D}$ be a $(P S)_{\delta}$ sequence of $I_{\lambda, \mu}$ for some $\delta \in \mathbb{R}$. Then,

$$
u_{n} \rightarrow u \quad \text { in } \mathscr{D}
$$

and either

$$
\begin{gathered}
u_{n} \longrightarrow u \\
\text { or } \delta \geq I_{\lambda, \mu}(u)+\left(\frac{(\gamma-2)}{2 \gamma}\right)\left(S_{\mu, \gamma}\right)^{\gamma /(\gamma-2)} .
\end{gathered}
$$

Proof. We know that $\left(u_{n}\right)$ is bounded in $\mathscr{D}$. Up to a subsequence if necessary, we have that

$$
\begin{aligned}
& u_{n} \rightarrow u \quad \text { in } \mathscr{D} \\
& u_{n} \longrightarrow u \text { a.e. in } \mathbb{R}^{N} .
\end{aligned}
$$

Denote $v_{n}=u_{n}-u$, and then $v_{n} \rightarrow 0$. As in Brézis and Lieb [16], we have

$$
\begin{aligned}
\int_{\mathbb{R}^{N}}|y|^{-b}\left|u_{n}\right|^{\gamma} & =\int_{\mathbb{R}^{N}}|y|^{-b}\left|v_{n}\right|^{\gamma}+\int_{\mathbb{R}^{N}}|y|^{-b}|u|^{\gamma}, \\
\left\|u_{n}\right\|_{\mu}^{2} & =\left\|v_{n}\right\|_{\mu}^{2}+\|u\|_{\mu}^{2} .
\end{aligned}
$$

From Lebesgue theorem and by using the assumption $(G)$, we obtain

$$
\begin{aligned}
& \lim _{n \rightarrow \infty} \int_{\mathbb{R}^{N}} g(x)|y|^{-b}\left|u_{n}\right|^{\gamma} d x \\
& \quad=\lim _{n \rightarrow \infty} \int_{\mathbb{R}^{N}} g(x)|y|^{-b}|u|^{\gamma} d x .
\end{aligned}
$$

Then, we deduce that

$$
\begin{aligned}
I_{\lambda, \mu}\left(u_{n}\right)= & I_{\lambda, \mu}(u)+\left(\frac{1}{2}\right)\left\|v_{n}\right\|_{\mu}^{2} \\
& -\left(\frac{1}{\gamma}\right) \int_{\mathbb{R}^{N}}|y|^{-b}\left|v_{n}\right|^{\gamma}+o_{n}(1), \\
\left\langle I_{\lambda, \mu}^{\prime}\left(u_{n}\right), u_{n}\right\rangle= & \left\|v_{n}\right\|_{\mu}^{2}-\int_{\mathbb{R}^{N}}|y|^{-b}\left|v_{n}\right|^{\gamma}+o_{n}(1) .
\end{aligned}
$$

From the fact that $v_{n} \rightarrow 0$ in $\mathscr{D}$, we can assume that

$$
\lim _{n \rightarrow \infty}\left\|v_{n}\right\|_{\mu}^{2}=\lim _{n \rightarrow \infty} \int_{\mathbb{R}^{N}}|y|^{-b}\left|v_{n}\right|^{\gamma}=\alpha \geq 0
$$

Assuming that $\alpha>0$, we have by definition of $S_{\mu, \gamma}$

$$
\alpha \geq S_{\mu, \gamma} l^{(2 / \gamma)}
$$

and so

$$
\alpha \geq\left(S_{\mu, \gamma}\right)^{\gamma /(\gamma-2)}
$$

Then, we get

$$
\delta \geq I_{\lambda, \mu}(u)+\left(\frac{(\gamma-2)}{2 \gamma}\right)\left(S_{\mu, \gamma}\right)^{\gamma /(\gamma-2)} .
$$

Therefore, if not, we obtain $\alpha=0$. That is, $u_{n} \rightarrow u$ in $\mathscr{D}$.

Lemma 5. Suppose that $2<k \leq N, \mu<\bar{\mu}_{k}$, and $(G)$ hold. If $(2, \gamma) \in \mathscr{R}_{1} \cup \mathscr{R}_{2}$, then there exist $\Lambda^{*}>0$ and $\varrho$ and $\nu$ positive constants such that, for all $\lambda>\Lambda^{*}$,

(i) there exist $\omega \in \mathbb{R}^{N}$ such that $I_{\lambda, \mu}(\omega)<0$,

(ii) we have

$$
I_{\lambda, \mu}(u) \geq v>0 \text { for }\|u\|_{\mu}=\varrho_{0} .
$$

Proof. (i) Let $t_{0}>0$ where $t_{0}$ is small, and $\phi \in C_{0}^{\infty}\left(\left(\mathbb{R}^{k} \backslash\right.\right.$ $\{0\}) \times \mathbb{R}^{N-k}$ ) such that $\phi \neq \equiv 0$. Choosing $\Lambda^{*}=\left|t_{0} \phi\right|^{1-\gamma}$, then, if $\lambda>\Lambda^{*}$ large enough,

$$
\begin{aligned}
I_{\lambda, \mu}\left(t_{0} \phi\right):= & \left(\frac{t_{0}^{2}}{2}\right)\|\phi\|_{\mu}^{2}-\left(\frac{t_{0}^{\gamma}}{\gamma}\right) \int_{\mathbb{R}^{N}}|y|^{-b}|\phi|^{\gamma} 1 \\
& -\left(\frac{t_{0}^{\gamma}}{\gamma}\right) \int_{\mathbb{R}^{N}}|y|^{-b}|\phi|^{\gamma} \lambda g(x) \\
< & \left(\frac{t_{0}^{2}}{2}\right)\|\phi\|_{\mu}^{2}-\left(\frac{t_{0}^{\gamma}}{\gamma}\right) \int_{\mathbb{R}^{N}}|y|^{-b}|\phi|^{\gamma} 1 \\
& -\left(\frac{t_{0}}{\gamma}\right) \int_{\mathbb{R}^{N}}|y|^{-b}|\phi| g(x)<0 .
\end{aligned}
$$

Thus, if $\omega=t_{0} \phi$, we obtain that $I_{\lambda, \mu}(\omega)<0$. 
(ii) By the Holder inequality and the definition of $S_{\mu, \gamma}$ and since $\gamma>2$, we get for all $u \in \mathscr{D} \backslash\{0\}$

$$
\begin{aligned}
I_{\lambda, \mu}(u):= & \left(\frac{1}{2}\right)\|u\|_{\mu}^{2} \\
& -\left(\frac{1}{\gamma}\right) \int_{\mathbb{R}^{N}}|y|^{-b}|u|^{\gamma}(1+\lambda g(x)) d x \\
\geq & \left(\frac{1}{2}\right)\|u\|_{\mu}^{2}-\left(\frac{1}{\gamma}\right) S_{\mu, \gamma}\|u\|_{\mu}^{\gamma}\left(1+\lambda|g|_{\infty}\right) .
\end{aligned}
$$

If $\lambda>\Lambda^{*}$, then there exist $\nu>0$ and $\varrho_{0}>0$ small enough such that

$$
I_{\lambda, \mu}(u) \geq \nu>0 \text { for }\|u\|_{\mu}=\varrho_{0}
$$

We also assume that $t_{0}$ is small enough such that $\left\|t_{0} \phi\right\|_{\mu}<\varrho_{0}$. Thus, we have

$$
\begin{aligned}
& c_{1}=\inf \left\{I_{\lambda, \mu}(u): u \in B_{\varrho_{0}}\right\}<0, \\
& \text { where } B_{\varrho_{0}}=\left\{u \in \mathscr{D}, \mathcal{N}(u) \leq \varrho_{0}\right\} .
\end{aligned}
$$

Using Ekeland's variational principle, for the complete metric space $\bar{B}_{\rho_{0}}$ with respect to the norm of $\mathscr{D}$, we can prove that there exists a $(P C)_{c_{1}}$ sequence $\left(u_{n}\right) \subset \bar{B}_{\rho_{0}}$ such that $u_{n} \rightarrow u_{1}$ for some $u_{1}$ with $\mathscr{N}\left(u_{1}\right) \leq \rho_{0}$.

Now, we claim that $u_{n} \rightarrow u_{1}$. If not, by Lemma 4 , we have

$$
\begin{aligned}
c_{1} & \geq I_{\lambda, \mu}\left(u_{1}\right)+\left(\frac{(\gamma-2)}{2 \gamma}\right)\left(S_{\mu, \gamma}\right)^{\gamma /(\gamma-2)} \\
& \geq c_{1}+\left(\frac{(\gamma-2)}{2 \gamma}\right)\left(S_{\mu, \gamma}\right)^{\gamma /(\gamma-2)}>c_{1},
\end{aligned}
$$

which is a contradiction.

Then, we obtain a critical point $u_{1}$ of $I_{\lambda, \mu}$ for all $\lambda>\Lambda^{*}$ large enough satisfying

$$
c_{1}=\left(\frac{(\gamma-2)}{2 \gamma}\right)\left\|u_{1}\right\|_{\mu}^{2}>0 .
$$

Proof of Theorem 1. From Lemmas 4 and 5, we can deduce that there exists at least a nontrivial solution $u_{1}$ for our problem $\left(\mathscr{P}_{\lambda, \mu}\right)$ with positive energy.

\section{Conflict of Interests}

The author declares that there is no conflict of interests regarding the publication of this paper.

\section{References}

[1] M. Badiale, V. Benci, and S. Rolando, "Solitary waves: physical aspects and mathematical results," Rendiconti del Seminario Matematico, vol. 62, no. 2, pp. 107-154, 2004.
[2] V. Benci and D. Fortunato, "Solitary waves in the nonlinear wave equation and in gauge theories," Journal of Fixed Point Theory and Applications, vol. 1, no. 1, pp. 61-86, 2007.

[3] W. A. Strauss, "Nonlinear invariant wave equations," in Invariant Wave Equations, vol. 73 of Lecture Notes in Physics, pp. 197249, Springer, Berlin, Germany, 1978.

[4] G. B. Withan, Linear and Nonlinear Waves, John Wiley \& Sons, 1974.

[5] M. Badiale, M. Guida, and S. Rolando, "Elliptic equations with decaying cylindrical potentials and power-type nonlinearity," Advances in Differential Equations, vol. 12, no. 12, pp. 1321-1362, 2007.

[6] P. Caldiroli and R. Musina, "On the existence of extremal functions for a weighted Sobolev embedding with critical exponent," Calculus of Variations and Partial Differential Equations, vol. 8, no. 4, pp. 365-387, 1999.

[7] F. Catrina and Z. Q. Wang, "On the Ca Caffarelli-KohnNirenberg inequalities: sharp constants, existence (and nonexistence), and symmetry of extremal functions," Communications on Pure and Applied Mathematics, vol. 54, no. 2, pp. 229-258, 2001.

[8] H. Brézis and L. Nirenberg, "Positive solutions of nonlinear elliptic equations involving critical sobolev exponents," Communications on Pure and Applied Mathematics, vol. 36, no. 4, pp. 437-477, 1983.

[9] B. Xuan, "The solvability of quasilinear Brezis-Nirenberg-type problems with singular weights," Nonlinear Analysis: Theory, Methods and Applications, vol. 62, no. 4, pp. 703-725, 2005.

[10] M. Badiale and S. Rolando, "A note on nonlinear elliptic problems with singular potentials, supported by MIUR, project," in Variational Methods and Nonlinear Differential Equations, 2005.

[11] R. Musina, "Existence of extremals for the Maz'ya and for the Caffarelli-Kohn-Nirenberg inequalities," Nonlinear Analysis, Theory, Methods and Applications, vol. 70, no. 8, pp. 30023007, 2009.

[12] I. Ekeland, "On the variational principle," Journal of Mathematical Analysis and Applications, vol. 47, no. 2, pp. 324-353, 1974.

[13] Adimurthi, N. Chaudhuri, and M. Ramaswamy, "An improved Hardy-Sobolev inequality and its application," Proceedings of the American Mathematical Society, vol. 130, no. 2, pp. 489-505, 2002.

[14] M. Gazzini and R. Musina, "Hardy-Sobolev-Maz'ya inequalities: symmetry and breaking symmetry of extremal functions," Communications in Contemporary Mathematics, vol. 11, no. 6, pp. 993-1007, 2009.

[15] L. Caffarelli, R. Kohn, and L. Nirenberg, "First order interpolation inequalities with weights," Compositio Mathematica, vol. 53, no. 3, pp. 259-275, 1984.

[16] H. Brézis and E. Lieb, "A relation between pointwise convergence of functions and convergence of functionals," Proceedings of the American Mathematical Society, vol. 88, no. 3, pp. 486486, 1983. 


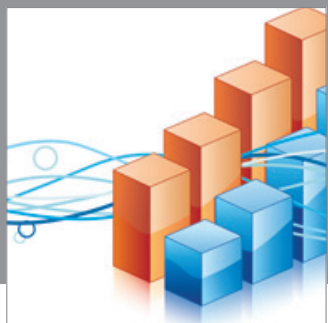

Advances in

Operations Research

mansans

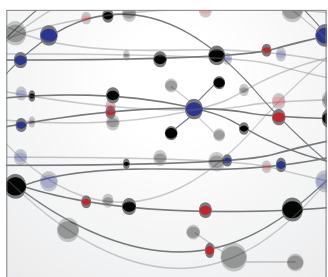

The Scientific World Journal
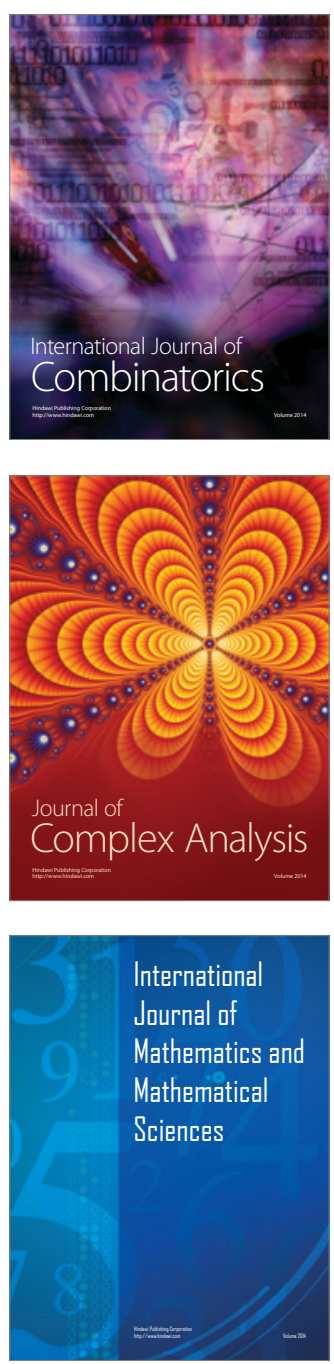
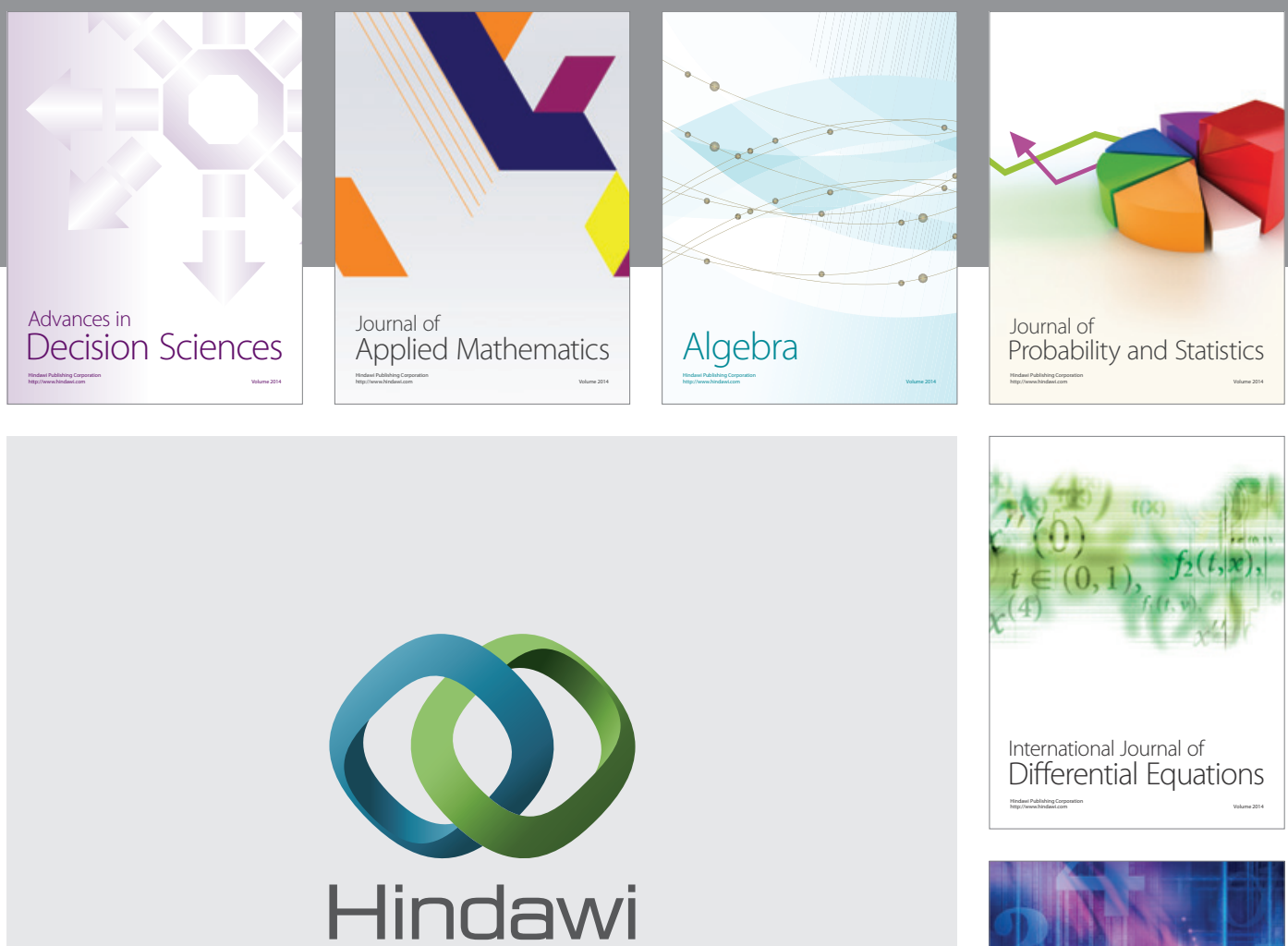

Submit your manuscripts at http://www.hindawi.com
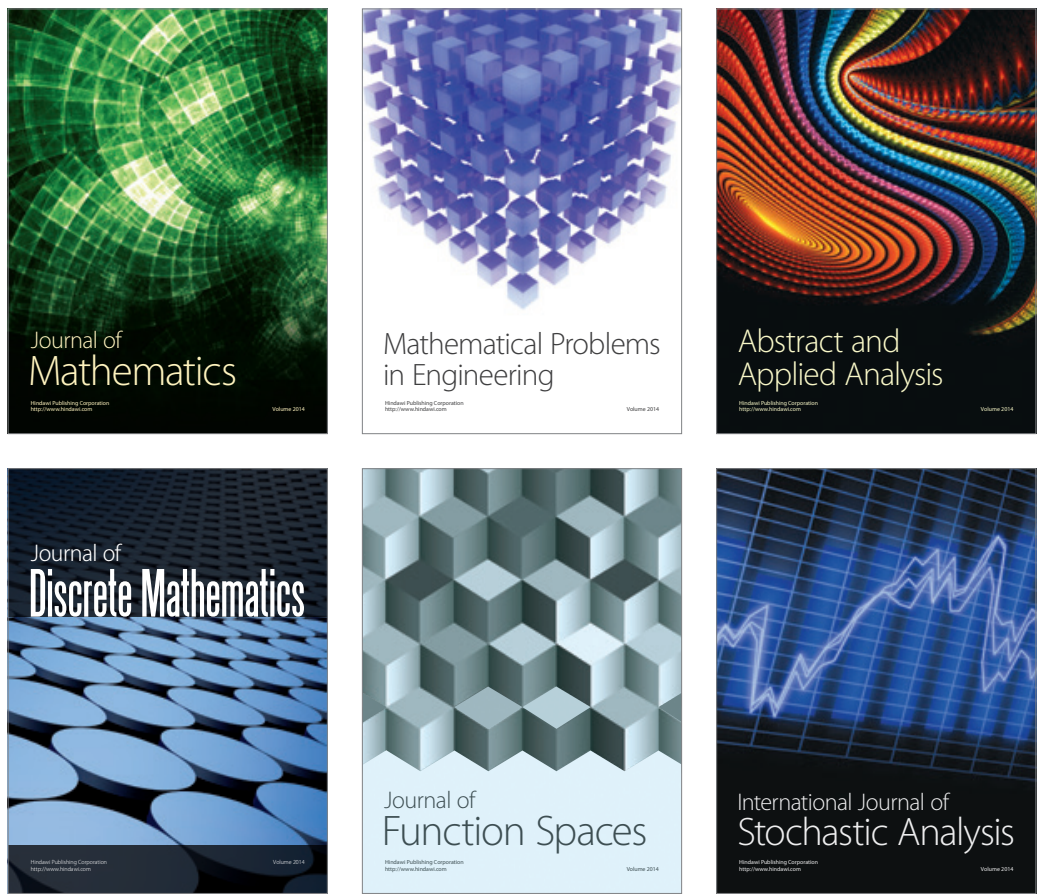

Journal of

Function Spaces

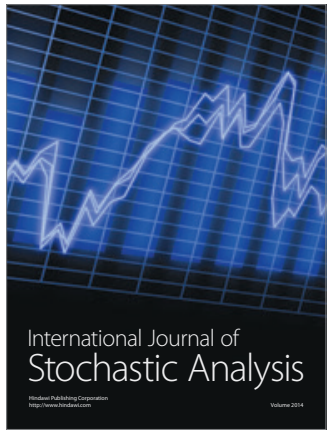

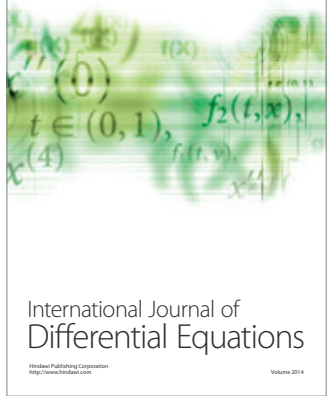
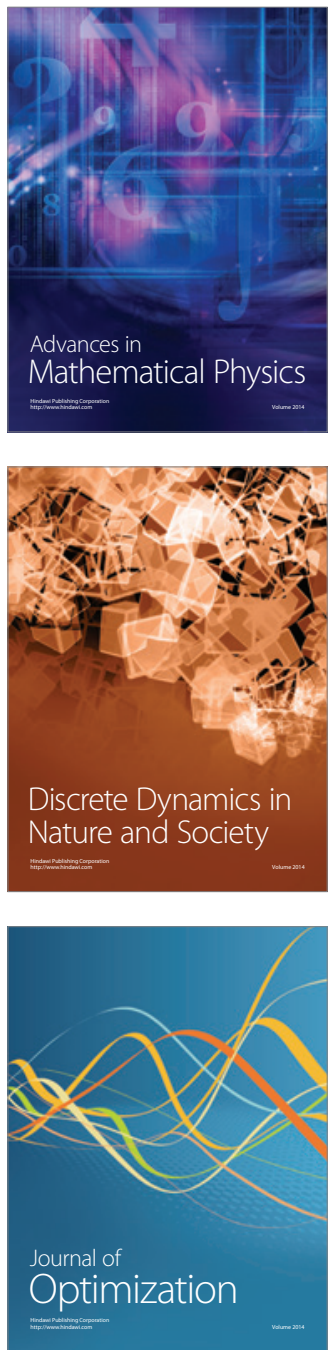